\title{
Paradox of HIV stigma in an integrated chronic disease care in rural South Africa: viewpoints of users and providers
}

Soter Ameh ( $\sim$ sote_ameh@yahoo.com )

University of Calabar https://orcid.org/0000-0002-8449-6423

Lucia D'Ambruoso

University of Aberdeen Institute of Applied Health Sciences

Francesc GOMEZ-OLIVE

University of the Witwatersrand

Kathleen Kahn

University of the Witwatersrand

Stephen Tollman

University of the Witwatersrand

Kerstin Klipstein-Grobusch

Universiteit Utrecht

Research article

Keywords: HIV stigma, integrated, chronic disease, primary health care, South Africa

Posted Date: December 11th, 2019

DOI: https://doi.org/10.21203/rs.2.18659/v1

License: (c) (1) This work is licensed under a Creative Commons Attribution 4.0 International License. Read Full License

Version of Record: A version of this preprint was published at PLOS ONE on July 31st, 2020. See the published version at https://doi.org/10.1371/journal.pone.0236270. 


\section{Abstract}

Background: An integrated chronic disease management (ICDM) model was introduced by the National Department of Health in South Africa to tackle the dual burden of HIV/AIDS and non-communicable diseases. One of the aims of the ICDM model is to reduce HIV-related stigma. There is a dearth of literature on reduction of HIV stigma attributable to the ICDM model. This paper describes the viewpoints of health care users and providers on HIV stigma in an ICDM model in rural South Africa.

Methods: A qualitative case study of HIV stigmatisation in the context of the implementation of an ICDM model in seven primary health care (PHC) facilities and their catchment communities was conducted in 2013 in the rural Agincourt sub-district, South Africa. Eight Focus Group Discussions were used to obtain data from 61 purposively selected participants who were 18 years and above. Seven In-Depth Interviews were conducted with the nurses-in-charge of the facilities. The transcripts were thematically analysed using MAXQDA 2018 qualitative software. The emerging themes on HIV stigma and HIV-related concerns were inductively analysed.

Results: Both service providers and users perceived implementation of the ICDM model may have led to reduced HIV stigma in the facilities. On the other hand, service users and providers thought HIV stigma increased in the communities because community members thought that home-based carers visited the homes of HIV-infected people. Service users thought that routine HIV testing, intended for pregnant women as stipulated in the World Health Organization guidelines, was associated with unwanted pregnancies among adolescents who wanted to use contraceptives but refused to take a HIV test as a precondition for receiving contraceptives. Caregivers of ill persons wanted full disclosures of the HIV status of their family members to enable them to protect their health and contribute to enhancing adherence to anti-retroviral therapy of HIV-infected family members.

Conclusions: Although the ICDM model was perceived to have contributed to reducing HIV stigma in the health facilities, it was associated with stigma in the communities. Remodelling the community component of the ICDM model could contribute to HIV stigma reduction in the study setting and elsewhere in South Africa.

\section{Background}

Human immune-deficiency virus (HIV) prevalence in South Africa was estimated at $13.1 \%$ in 2018 , one of the highest in Africa (1). Approximately 7.52 million people lived with HIV in 2018, with adults 1549 years having a higher prevalence of $19 \%$ than the general population [1]. The HIV burden in South Africa is unequal by race, age and sex. The prevalence in black populations is $40-50$ times that of white (2) and age-adjusted HIV prevalence differs significantly between women (26\%) and men (19\%) (3). In adolescents, the risks of infection are eight times higher in females than males (2). One of the main challenges People Living with HIV (PLWH) face is stigmatisation which is considered to occur at three levels: enacted, anticipated and internalized. Enacted stigma occurs when PLWH believe they experience 
prejudice and discrimination $(4,5)$. Anticipated stigma occurs when PLWH have an expectation that they will experience prejudice and discrimination (6). Internalized stigma refers to a situation where PLWH endorse the negative beliefs and feelings associated with HIV/AIDS (5).

HIV stigmatisation could negatively impact achievement of the zero discrimination and 95-95-95 (i.e. 95\% of people who live with HIV knowing their status, $95 \%$ of people who know their status receiving treatment and $95 \%$ of people on HIV treatment having a suppressed viral load) targets of ending the HIV/AIDS epidemic by 2030 in the context of the Sustainable Development Goals (SDGs) (7). However, a study in Cambodia showed that an integrated care for HIV/AIDS, hypertension and diabetes can act as an impetus to reduce HIV stigma (8).

To address HIV stigmatisation in the context of the dual burden of HIV and chronic non-communicable disease (NCDs) in South Africa $(9,10)$, the National Department of Health in 2011 introduced an Integrated Chronic Disease Management (ICDM) model as a pilot intervention programme in primary health care (PHC) facilities using the health systems approach $(11,12)$. The purpose of the ICDM model of care is to leverage the HIV vertical programme to support or scale up services for NCDs to reduce HIV stigmatisation and to improve health outcomes of patients with NCDs (8). The model has a facility component in which facilities are reorganised to enable providers to offer services for HIV and NCDs in a 'one-stop-shop' in designated chronic care areas and a community component in which a primary health care (PHC) outreach team, made up of a nurse and community health care workers, visit patients' homes to provide home-based care and link clinic defaulters back to care (13).

Little is known about HIV stigmatisation in the context of the ICDM model of care. The aim of this research was to assess HIV stigma from the viewpoints of health care users and providers in a rural setting in South Africa.

\section{Methods}

\section{Study site}

The site for this study was in the Agincourt sub-district, Mpumalanga Province, South Africa. During commencement of data collection (August to October 2013), ICDM was being implemented in 17 of the $38 \mathrm{PHC}$ facilities in the municipality. Seven of the $17 \mathrm{PHC}$ facilities were selected because they are situated in the Ehlanzeni health district where the Medical Research Council/Wits Agincourt Research Unit has surveyed the population since 1992 using a Health and Demographic Surveillance System (HDSS). As of July 2011, the population under surveillance in the Agincourt HDSS was 90,000 people in 16,000 households living in 27 villages (14).

\section{Study design and population}

This qualitative research is a case study of implementation of an ICDM model in PHC facilities and their catchment communities, and a part of a broader mixed methods research project which evaluated the 
quality of care in the ICDM model (15) in the study setting. The study population consisted of clients 18 years and above being managed for HIV, hypertension and diabetes in the seven PHCs in the subdistrict. The Facility Managers (i.e. professional nurses-in-charge of the seven facilities) were also considered part of the study population because of their viewpoints as service providers and facility managers.

\section{Inclusion and exclusion criteria}

Patients diagnosed with and being managed in the health facilities for HIV, hypertension, and diabetes were eligible to participate in the study. Inclusion criteria were being on treatment six months before the ICDM model was implemented, participating in health facility exit quantitative interviews on quality of the ICDM model of care commenced in June 2013 (15) and willingness to participate in the current Focus Group Discussions (FGDs). The managers of the seven health facilities in the district were purposively selected for the In-depth Interviews (IDIs) based on their depth of experience.

\section{Data collection}

The exit interviews provided a large sampling frame from which purposive selection of prospective participants for the FGDs was done to explore patients' in-depth perspectives on the quality of care in the ICDM model. During the exit interview of 435 randomly selected patients in the quantitative study on quality of care in the ICDM model (15), 80 patients who volunteered to participate in the FGDs were invited to do so based on the inclusion criteria described above. Of the 80 purposively selected prospective participants, 61 participated in the eight FGDs (one FGD per health facility and one FGD for defaulters, defined as those who missed three or more consecutive clinic appointments as was observed through the review of clinic records) giving a response rate of $76 \%$.

The prospective FGD participants were recruited from the seven PHC facilities during clinic opening hours on week days. The FGDs were then held on Saturdays at a convenient time for most of the patients and in generally centrally located designated venues within the catchment communities served by the seven health facilities. The FGDs were held outside facilities to encourage participants to freely express and communicate their lived experiences of HIV stigma without fear of intimidation or victimisation. Two qualitative fieldworkers conducted the FGDs with 5-9 participants of similar age with each session lasting 60-90 minutes. The more senior field worker moderated the discussions which were held in Tsonga language and with permission were audiotaped while the second facilitator assisted and took notes.

Seven IDIs were conducted with the nurses-in-charge of the seven health facilities. The Principal Investigator (PI, S.A.) conducted and audiotaped the IDIs with the facility managers in English. The IDIs were held in the offices of the facility managers during lunch break with an average duration of 30 minutes.

\section{Quality assurance}


The PI had a two-day training session with two senior qualitative field workers who had worked in the Agincourt HDSS for at least 10 years. The PI briefed field workers about the purpose of the study and discussions were held on how to administer the topic guide and facilitate the discussions encouraging participation and balanced, coherent discussion. The audio recordings of the FGDs were translated and transcribed into English by the two qualitative field workers. A third qualitative field worker, who was blinded to the other two field workers, validated the transcriptions by listening to two of the seven audiotapes and translating them into English. There were no major differences between the first and second transcriptions. A similar procedure was used to assure data quality for the IDIs.

\section{Data analysis}

The transcribed FGDs and IDIs were thematically analysed using MAXQDA 2018 qualitative software. The emerging theme on HIV stigma and HIV-related concerns were inductively analysed. The PI coded the data and developed a codebook. The codes were verified by the co-authors through the reading and rereading of the quotes. This paper focuses on the theme on HIV stigma and HIV-related matters.

\section{Results}

The viewpoints of healthcare users and providers on HIV stigma and HIV related concerns are presented below. Verbatim quotes from users and providers are used to illustrate the main HIV theme and subthemes on enacted, anticipated and internalised domains of stigma.

Socio-demographic characteristics of the study_population

Sixty-one (61) black adult South Africans (43 females and 18 males) participated in the eight FGDs. The higher number of female than male participants reflects the female: male ratio of 7:3 in a populationbased study conducted earlier in the study setting (16). All the seven facility managers were females aged 40 to 55 years.

\section{Reduced HIV stigma in the health facilities}

A facility manager, whose views represented that of many managers, reported that the ICDM model of care reduced HIV stigma by other clients due to non-segregation of patients managed for chronic diseases in the same clinic. This was because the former practice of segregating patients according to the illness they were being managed for made it easy to identify who was receiving treatment for HIV/AIDS in the health facilities.

Previously we were grouping them [patients] according to their diseases, but now they are put together. Patients living with HIV/AIDS are satisfied because they are mixed with those who are having hypertension and diabetes [IDI with Manager, Health Facility 6].

A woman described how she did not fear being identified as a patient attending a follow-up clinic to receive anti-retroviral treatment because professional nurses treated hypertension and diabetes patients 
as well as PLWH in the same consultation room. This view was expressed by participants from some other health facilities.

In the past, there used to be a separate clinic for HIV patients. But now, all of us (referring to chronic disease patients) are getting our medication in one room. It is not easy for people [referring to other clients] to say I am HIV positive [Respondent 1 (woman), FGD Health Facility 5].

\section{$\underline{\text { Stigma in home-based care }}$}

Facility managers recounted how some community members stigmatised ill people who were visited in their homes by home-based carers (HBCs). These community members were said to have perceived persons visited by HBCs to have HIV/AIDS, and that patients responded by not allowing HBCs to visit their homes because they thought that HBCs divulged their personal information to some community members (anticipated and internalised stigma). The implication of this is that activities of HBCs could negatively impact implementation of the ICDM model.

I told them not to come to my house any more. When I tell them something I expect them to report it to their seniors not to tell the whole community [Respondent 5 (woman), FGD for Health Facility 2].

Home-based carers are not accepted. They [PLWH] are thinking that other people [community members] will think that they are HIV positive and that is why the HBCs are not allowed to visit them at home [IDI with Manager, Health Facility 1].

\section{Stigmatisation by nurses}

It was recounted in some FGDs how interactions between nurses and HIV positive women who visited the facility to utilise antenatal care services showed an expectation that clients on ART should not get pregnant or should not desire to have children because of their HIV positive status. This practice reinforces enacted stigma and could be a barrier to reproductive health care.

I gave my file that I am using to take treatment [referring to ART] and the other file for pregnancy [referring to antenatal care] and she [referring to a nurse] says: are you pregnant again? She said it in a bad way. Then I asked her why she is talking like that and she said that a person like me is not supposed to get pregnant. Then I said to her: if I am HIV positive, does it mean I am not supposed to have children? I know my status and I know how to take care of myself. I didn't feel okay when she ask me that why am I pregnant. It means when a person is positive she is not supposed to get pregnant? It means I am different from other people? [Respondent 6 (woman) FGD for Health Facility 3].

Routine HIV testing as a barrier to contraceptive use and family_planning

Respondents reported that young women who visited facilities for the purposes of receiving contraceptives or family planning were compelled to take a HIV test, even though the national contraception and family planning policy did not prescribe such practice. Clients who refused to take a 
HIV test were denied access to contraceptives or family planning; hence, routine HIV testing was experienced as a barrier to uptake of reproductive health services.

What I have observed is that, there are illnesses that you have to volunteer to be tested when you go to the clinic. But when young women go to the clinic for contraceptives, they are forced to test for HIV whether they like it or not. If you refuse to test you don't get what you were there for [Respondent 3 (woman), FGD for Health Facility 1]

Some participants in the FGDs recounted how young girls who were denied access to contraceptives because they refused to be tested for HIV by nurses in the health facilities were at risk of getting pregnant, and how pregnancy resulting from denial in access to contraceptives as a result of refusal to take a routine HIV test was associated with or seemed to exacerbate poverty.

At the clinic, when they go for contraceptives, they [nurses] don't give them [to clients] without testing for HIV. The young girls refuse to test and they will go home without getting the contraceptives. When you see lots of young women being pregnant, it is because they are afraid to test for HIV. And when they get pregnant they are creating poverty in their families but if she goes and tests, she will get the contraceptives that she wants [Respondent 3 (woman) FGD for Health Facility 2].

HIV testing and disclosure of patients' HIV status to family members

Respondents expressed views that HIV testing and disclosure of HIV status of a sick family member would enable parents or caregivers to provide care and support services to ensure adherence to medication. Furthermore, such disclosure could equip family members with the knowledge to take preventive measures from being infected with HIV.

You find a person [family member] being told by the nurse that she has HIV, but she [patient] will not tell the family that she is having HIV. She will get sicker and she will not tell you and you will go around to the clinics and hospital trying to find a cure for her meanwhile she knows what is killing her [Respondent 3 (woman) FGD for Health Facility 2].

I once went to the clinic with my cousin and she refused to be tested, I told her that she has to test whether she likes it or not, and the nurses said to me that I must not force her because it's not according to the law. I told them that she is sick and I am the one to take care of her, how am I going to do it without knowing what is eating her. So we have to fight them until they get tested [Respondent 3 (woman) FGD for Health Facility 2].

\section{Discussion}

This study showed convergent viewpoints of service users and providers on the ICDM model being associated with reduced HIV stigma in the health because clients managed for HIV and NCDs were mixed together in the waiting and consultation rooms. On the other hand, both users and providers also had convergent perceptions of HIV stigmatisation in the communities which were related to the recounted 
practices of HBCs visiting the homes of PLWH. Other findings, from the viewpoints of users, suggested nurses stigmatised HIV positive pregnant women for being pregnant; and conducted routine HIV testing of non-pregnant adolescents who wanted to use contraceptives; hence, unwanted pregnancy was an unintended consequence of non-use of contraceptives due to adolescents' refusal to take a routine HIV test. Finally, caregivers wanted HIV testing and/or disclosure of HIV status of sick family members which they perceived was an enabler for them to support PLWH to adhere to ART and to take preventive measures from being infected with HIV.

The pattern of HIV stigmatisation observed in this study is a paradox. On the one hand, the ICDM model was associated with HIV stigma reduction in PHC facilities in the study setting, as has been reported in Cambodia where HIV care was integrated with those of hypertension and diabetes in designated hospitals (8). However, the model was related to HIV stigmatisation in the communities where HBCs traced clients who defaulted from the clinics for the purposes of linking them back to care. During such visits, community members identified the homes visited by HBCs and perceived that the persons being visited were PLWH. This may imply that the design of the community-based component of the ICDM model may not have sufficiently addressed HIV stigmatisation at the community level. As shown elsewhere in South Africa (17), lessons learnt from a HIV stigma-reduction community "hub" intervention could be useful in mobilising and educating HBCs and community members towards HIV stigma reduction in the study setting.

Although the ICDM model of care was associated with reduced HIV stigma in the health facilities, the perspective of service users was that enacted stigma occurred among pregnant HIV positive women who visited the antenatal clinic. These women felt stigmatised when nurses told them they were not supposed to get pregnant because of their HIV status. Aside stigma, the nurses may have intended for the pregnant women to have lower reproductive intention, which is associated with HIV positive status. This has been reported in a study that used National Demographic and Health Surveys to determine the effect of HIV status on fertility intention among women in nine sub-Saharan African countries. The study showed that women who were HIV positive, with knowledge of their status, had lower odds of wanting more children (18). Anticipated and internalised stigmas were experienced by PLWH in the communities when HBCs visited their homes which were identifiable by community members. This could have negative implications for activities of HBCs in implementing the community component of the ICDM model.

Routine HIV testing for adolescents in the study setting was perceived to be a barrier to uptake of reproductive health services and contravened South Africa's Children's Act (19). Section 134 of the Act facilitates children's access to contraception with the aim of preventing sexually active children $\geq$ 12 years from contracting sexually transmitted infections (including HIV) or falling pregnant (19). Routine HIV testing, which is intended for pregnant women, has its origin in the guideline on HIV testing established by the World Health Organization (WHO) in 2004 (20). The guideline recommends routine HIV testing for all pregnancy-related visits by healthcare providers, especially in high HIV transmission areas with the intention of reducing mother-to-child HIV transmission. This study showed evidence suggestive of health workers' misapplication of the WHO guideline, through refusal to provide contraceptives to 
adolescents who declined to take an HIV test, led to unintended consequences such as unwanted pregnancy which in turn was viewed as a key influence on poverty exacerbation.

The WHO guidelines for HIV testing and counselling for adolescents living with HIV recommend early and full disclosure of HIV status of children of school age as this improves ART adherence(21). The findings of this study suggests that parents or guardians wanted routine HIV testing and/or disclosures of the HIV status of their adolescent wards when they were ill. Parents thought this would enable them to take preventive measures from being infected with HIV, provide care and ensure adherence to ART. This is corroborated in a study in South Africa that showed that early and full disclosure is strongly associated with improved adherence amongst ART-initiated adolescents 10-19 years of age; hence, disclosure may be an essential tool in improving adolescent adherence and reducing mortality and onwards transmission (22).

\section{Conclusions}

Application of a comprehensive ICDM model was associated with reduced HIV stigma in the health facilities, but was linked to stigmatisation in the communities. Routine HIV testing, originally intended for pregnant women, of non-pregnant adolescents who desired to use contraceptives was related to unwanted pregnancies. Refusal to provide contraceptives to adolescents who declined to take an HIV test could have far reaching implications for adolescent-friendly reproductive health services. Caregivers of PLWH wanted routine HIV testing and/or disclosures of the HIV status of their family members for the purposes of protecting their own health and enhancing adherence to ART.

\section{Declarations}

\section{Ethics approval and consent to participate}

All procedures performed in studies involving human participants were in accordance with the ethical standards of the institutional and/or national research committee (University of the Witwatersrand, Johannesburg (M120943) and the Mpumalanga Provincial Research and Ethics Committee, South Africa.) and with the 1964 Helsinki declaration and its later amendments or comparable ethical standards. Informed consent was obtained from all individual participants included in the study.

\section{Consent for publication}

Not applicable.

\section{Availability of data and materials}

The datasets generated and/or analysed during the current study are not publicly available due to the need to protect the identity of the patients and health facilities in which the study was conducted as stated in the informed consent, but are available from the corresponding author on reasonable request. 


\section{Competing interests}

The authors declare that they have no competing interests.

\section{Funding}

The fieldwork for this research was funded by the following: 1) The Agincourt Health and SocioDemographic Surveillance System, a node of the South African Population Research Infrastructure Network (SAPRIN) and is supported by the National Department of Science and Innovation, the Medical Research Council and the University of the Witwatersrand, South Africa, and the Wellcome Trust, UK (grants 058893/Z/99/A; 069683/Z/02/Z; 085477/Z/08/Z; 085477/B/08/Z); 2) Fogarty International Centre of the National Institutes of Health under the award number D43 TW008330; and 3) an African Doctoral Dissertation Research Fellowship Programme award to the corresponding author. The content is solely the responsibility of the authors and does not necessarily represent the official view of the funders. The article processing charge for this manuscript was funded by the Lown Scholars Program in Cardiovascular Health, Department of Global Health and Population, Harvard T.H. Chan School of Public Health, Boston, Massachusetts, U.S.A.

\section{Authors' contributions}

SA, XFO, KKG, ST, KK and LD conceived the study. SA collected the data and coded them and all authors verified the codes. SA drafted the manuscript and all authors read and approved the final manuscript.

\section{Acknowledgements}

The authors acknowledge the contributions of Latonya Wilson for writing support.

\section{Abbreviations}

List of abbreviations 


\begin{tabular}{|ll|}
\hline Abbreviation & Full meaning \\
\hline ART & Antiretroviral Therapy \\
\hline FGDs & Focus Group Discussions \\
\hline HBCs & Home-based Carers \\
\hline HDSS & Health and Socio-Demographic Surveillance System \\
\hline HIV/AIDS & Human Immunodeficiency Virus/Acquired Immune Deficiency Syndrome \\
\hline ICDM & Integrated Chronic Disease Management \\
\hline IDIs & In-Depth Interviews \\
\hline NCDs & Non-communicable Diseases \\
\hline PHC & Primary Health Care \\
\hline PI & Principal Investigator \\
\hline PLWH & People Living With HIV/AIDS \\
\hline SDGs & Sustainable Development Goals \\
\hline WHO & World Health Organization \\
\hline
\end{tabular}

\section{References}

1. Statistics South Africa. 2018. Mid-year population estimates. https://www.statssa.gov.za/publications/P0302/P03022018.pdf. Accessed 20 Jul 2019.

2. Zuma K, Shisana O, Rehle TM, Simbayi LC, Jooste S, Zungu N, et al. New insights into HIV epidemic in South Africa: key findings from the National HIV Prevalence, Incidence and Behaviour Survey. Afr J AIDS Res. 2016;15 Suppl 1:67-75.

3. Clark SJ, Gomez-Olive FX, Houle B, Thorogood M, Klipstein-Grobusch K, Angotti N, et al. Cardiometabolic disease risk and HIV status in rural South Africa: establishing a baseline. BMC Public Health. 2015;15:135.

4. Valenzuela C, Ugarte-Gil C, Paz J, Echevarria J, Gotuzzo E, Vermund SH, et al. HIV stigma as a barrier to retention in HIV care at a general hospital in Lima, Peru: a case-control study. AIDS Behav. 2015;19 Suppl 2:235-245.

5. Sorsdahl KR, Mall S, Stein DJ, Joska JA. The prevalence and predictors of stigma amongst people living with HIV/AIDS in the Western Province. AIDS Care. 2011;23 Suppl 6:680-685.

6. Kingori C, Reece M, Obeng S, Murray M, Shacham E, Dodge B, et al. Psychometric evaluation of a cross-culturally adapted felt stigma questionnaire among people living with HIV in Kenya. AIDS Patient Care STDS 2013;27 Suppl 8:481-488. 
7. United Nation. Sustainable Development Goals.

https://www.un.org/sustainabledevelopment/health/. Accessed 30 Jun 2015.

8. Janssens B, Van Damme W, Raleigh B, Gupta J, Khem S, Soy Ty K, et al. Offering integrated care for HIV/AIDS, diabetes and hypertension within chronic disease clinics in Cambodia. Bull World Health Organ. 2007;85 Suppl 11:880-885.

9. Mayosi BM, Flisher AJ, Lalloo UG, Sitas F, Tollman SM, Bradshaw D. The burden of noncommunicable diseases in South Africa. Lancet. 2009;374 Suppl 9693:934-947.

10. Tollman SM, Kahn K, Sartorius B, Collinson MA, Clark SJ. Implications of mortality transition for primary health care in rural South Africa: a population-based surveillance study. Lancet. 2008;372.

11. Mahomed $\mathrm{OH}$, Asmall S. Development and implementation of an integrated chronic disease model in South Africa: lessons in the management of change through improving the quality of clinical practice. International Journal of Integrated Care. 2015;15:e038.

12. Mahomed $\mathrm{OH}$, Asmall S, Freeman $\mathrm{M}$. An integrated chronic disease management model: a diagonal approach to health system strengthening in South Africa. J Health Care Poor Underserved. 2014;25 Suppl 4:1723-1729.

13. Mahomed $\mathrm{OH}$, Asmall $\mathrm{S}$, Voce A. Sustainability of the integrated chronic disease management model at primary care clinics in South Africa. African Journal of Primary Health Care \& Family Medicine. 2016;8 Suppl 1:1248.

14. Kahn K, Collinson MA, Gomez-Olive FX, Mokoena O, Twine R, Mee P, et al. Profile: Agincourt health and socio-demographic surveillance system. Int J Epidemiol. 2012;41 Suppl 4:988-1001.

15. Ameh S, Gómez-Olivé FX, Kahn K, Tollman SM, Klipstein-Grobusch K. Relationships between structure, process and outcome to assess quality of integrated chronic disease management in a rural South African setting: applying a structural equation model. BMC Health Serv Res. 2017;17 Suppl 1:229.

16. Ameh S, Gomez-Olive FX, Kahn K, Tollman SM, Klipstein-Grobusch K. Predictors of health care use by adults 50 years and over in a rural South African setting. Glob Health Action. 2014;7:24771.

17. Prinsloo CD, Greeff M, Kruger A, Khumalo IP. HIV stigma experiences and stigmatisation before and after a HIV stigma-reduction community "hub" intervention. Afr J AIDS Res. 2017;16 Suppl 3:203-213.

18. Mumah JN, Ziraba AK, Sidze EM. Effect of HIV status on fertility intention and contraceptive use among women in nine sub-Saharan African countries: evidence from Demographic and Health Surveys. Glob Health Action. 2014;7:25579.

19. Department of Health, Republic of South Africa. National Contraception and Fertility Planning Policy and Service Delivery Guidelines. A companion to the National Contraception Clinical Guidelines, 2012. http://www.partners-popdev.org/wp-content/uploads/2015/08/National-contraception-familyplanning-policy.pdf. Accessed 10 Aug 2019.

20. The Joint United Nations Programme on HIV/AIDS /World Health Organization. UNAIDS/WHO Policy Statement on HIV Testing (2004). https://www.who.int/hiv/pub/vct/statement/en/. Accessed Jul 07 2019. 
21. World Health Organization. HIV and adolescents: Guidance for HIV testing and counselling and care for adolescents living with HIV 2013.

https://apps.who.int/iris/bitstream/handle/10665/94334/9789241506168_eng.pdf?sequence=1. Accessed Jul 2019.

22. Cluver LD, Hodes RJ, Toska E, Kidia KK, Orkin FM, Sherr L, et al. 'HIV is like a tsotsi. ARVs are your guns': associations between HIV-disclosure and adherence to antiretroviral treatment among adolescents in South Africa. AIDS. 2015;29 Suppl I 1:S57-65. 